\title{
Nuevo registro de Sphagnum falcatulum Besch (Sphagnaceae) en Isla Navarino, Reserva de la Biósfera Cabo de Hornos
}

\author{
New record of Sphagnum falcatulum Besch (Sphagnaceae) \\ on Navarino Island, Cape Horn Biosphere Reserve
}

Roy Mackenzie ${ }^{1,2}$, Lily R. Lewis ${ }^{1,3}$ \& Ricardo Rozzi ${ }^{1,4}$

En el extremo sur del continente sudamericano, en la ecorregión subantártica de Magallanes, se ubica la Reserva de la Biosfera Cabo de Hornos (Rozzi et al. 2006), en donde aún existe un bajo nivel de fragmentación de los ecosistemas y escasa población humana, por lo que la región se encuentra catalogada como una de las últimas 24 áreas prístinas del planeta (Mittermeier et al. 2003). En ésta, se ha registrado una alta diversidad de briófitas, por lo que ha sido identificada como un "hotspot" de biodiversidad de plantas no vasculares (Rozzi et al. 2008a). Sin embargo, en el contexto del cambio climático global, es indispensable continuar con los estudios a largo plazo sobre esta brioflora en este verdadero laboratorio natural (Goffinet et al. 2012).

Para contribuir a los estudios y conservación de las briófitas, el Parque Etnobotánico Omora, ubicado en la Isla Navarino, ha desarrollado un programa educativo de Conservación Biocultural con el método de Filosofía Ambiental de Campo. Esta aproximación combina las ciencias y la filosofía en el desarrollo de temáticas y actividades educativas y ecoturísticas que transformen la visión de los estudiantes y visitantes desde el simple placer y satisfacción de visitar una reserva de la biósfera, hacia una comprensión de las relaciones ecológicas y un cambio de actitud para fomentar un comportamiento más responsable con el medio ambiente.

Con el fin de caracterizar los hábitats de la Isla Navarino, en base a imágenes satelitales y verificaciones de campo, cuantificamos la cobertura de los tres tipos principales de ambientes terrestres identificados por Pisano (1977, 1992): bosques subantárticos (46\%), complejo de tundra (turbera) de Magallanes (36\%) y formaciones Altoandinas (18\%) (Fig. 1). Al mismo tiempo analizamos la proporción de especies, géneros y familias de musgos registrados para cada uno de estos tipos de hábitat en la Isla Navarino (Fig. 2). Llama la atención que menos del $20 \%$ de los taxa de musgos han sido registrados en el complejo de tundra de Magallanes que ocupa un $36 \%$ de la superficie de la Isla. La baja riqueza de briófitas registradas en este hábitat podría deberse a un menor esfuerzo de exploración briológica en turberas y a la carencia histórica de briólogos en la región de Magallanes. Por tanto, un esfuerzo de muestreo mayor en turberas podría contribuir al registro de especies con distribuciones biogeográficas desconocidas o incluso de nuevas especies endémicas.

Con el fin de estudiar en mayor profundidad la flora briológica asociada a turberas, en enero-

\footnotetext{
1 Instituto de Ecología y Biodiversidad \& Universidad de Magallanes, Parque Etnobotánico Omora, Puerto Williams, Chile.

2 Departamento de Genética Molecular y Microbiología, Facultad de Ciencias Biológicas, Pontificia Universidad Católica de Chile, Santiago, Chile. $\$ romackenzie@uc.cl

3 University of Florida, Department of Biology PO Box 118525 Gainesville FL 3261, USA. lilyrlewis@gmail.com

4 Sub-Antarctic Biocultural Conservation Program, University of North Texas, Denton, TX, USA. rozzi@unt.edu
} 


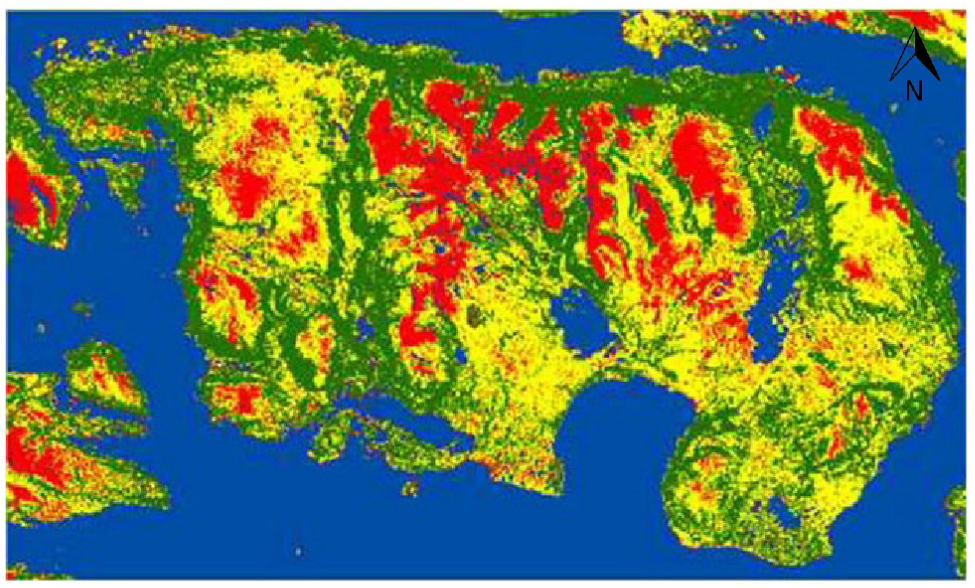

Altoandino
Bosque Subantártico

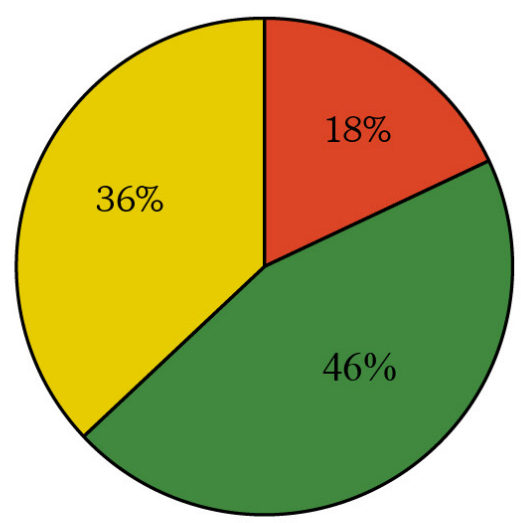

Turbera

Fig. 1. Cobertura de los principales tipos de hábitats en la isla Navarino: bosques subantárticos, ambientes altoandinos y complejo de tundra de Magallanes. Basada en imágenes satelitales Landsat 7 (año 2002) y SPOT 5 (año 2003) clasificación supervisada y verificaciones de campo realizadas por Sanja Fistric (Helmholtz-Zentrum für Umweltforschung GmbH - UFZ, Leipzig, Alemania), Rosa María Gallardo (Centro de Energías Renovables,

CERE, Universidad de Magallanes, UMAG) y Ricardo Rozzi.

febrero 2016 se realizó una expedición, atravesando la Isla Navarino de norte a sur desde Puerto Williams hasta el lago Windhond. En el sector sur de la Isla el paisaje está dominado por hábitats de turberas. De acuerdo a los primeros trabajos realizados en la zona por Pisano (1977 y 1992) y Domínguez et al. (2015), el complejo de tundra de Magallanes está compuesto por tres tipos principales de turberas: 1) Graminiformes, humedales de junquillar; 2) Pulviniformes, con plantas que crecen formando un cojín compacto; y 3) Turberas de Sphagnum. Los dos primeros hábitats están dominados por plantas vasculares: junquillar Marsippospermum grandiflorum (L.f.) Hook. (1843) y plantas en cojín de los géneros Astelia, Azorella, Donatia y Bolax. En cambio, las turberas están dominadas por plantas no-vasculares, particularmente por dos especies de musgo: Sphagnum magellanicum Brid. (1798) y S. fimbriatum Wilson (1847). En las turberas de los sectores sur de Isla Navarino confirmamos la dominancia de las especies $S$. fimbriatum y $S$. magellanicum descritas en estudios anteriores. Sin embargo, en la turbera pulviniforme (dominada por plantas en cojín, sensu Pisano 1980) donde se encuentran además abundantes poblaciones de especies de plantas carnívoras de los géneros Drosera y Pinguicula
(Fig. 3A) creciendo entre centenares de pequeñas pozas de agua de profundidad variable (entre 30 y $80 \mathrm{~cm}$ de profundidad, aproximadamente) con un sedimento rojizo (Fig. 3B), realizamos un nuevo registro de una especie de Sphagnum que ha sido escasamente registrada en la Isla Navarino: Sphagnum falcatulum Besch. (1885).

Los musgos del género Sphagnum son especies clave en las turberas donde a través de atributos morfológicos y bioquímicos generan un ambiente ácido, permeable y pobre en nutrientes (Van Breemen, 1995). Recientemente se ha descubierto que este ambiente inhóspito para la mayoría de las plantas vasculares es generado por Sphagnum asociado a una rica comunidad de microbios, o microbioma, que viven dentro (endosfera) o fuera (ectosfera) de los musgos (Kostka et al. 2016). Por lo tanto, los registros de nuevas poblaciones de Sphagnum falcatulum podrían conducir a futuros registros de nuevas especies microbianas. Se determinó que el agua en las pozas donde se registraron abundantes poblaciones de $S$. falcatulum de hecho tiene un $\mathrm{pH}$ marcadamente ácido de 4,67. Cerca de las orillas de estas pozas las poblaciones de $S$. falcatulum crecen completamente sumergidas bajo el agua (Fig. 3C), en contraste con los registros más comunes de las 


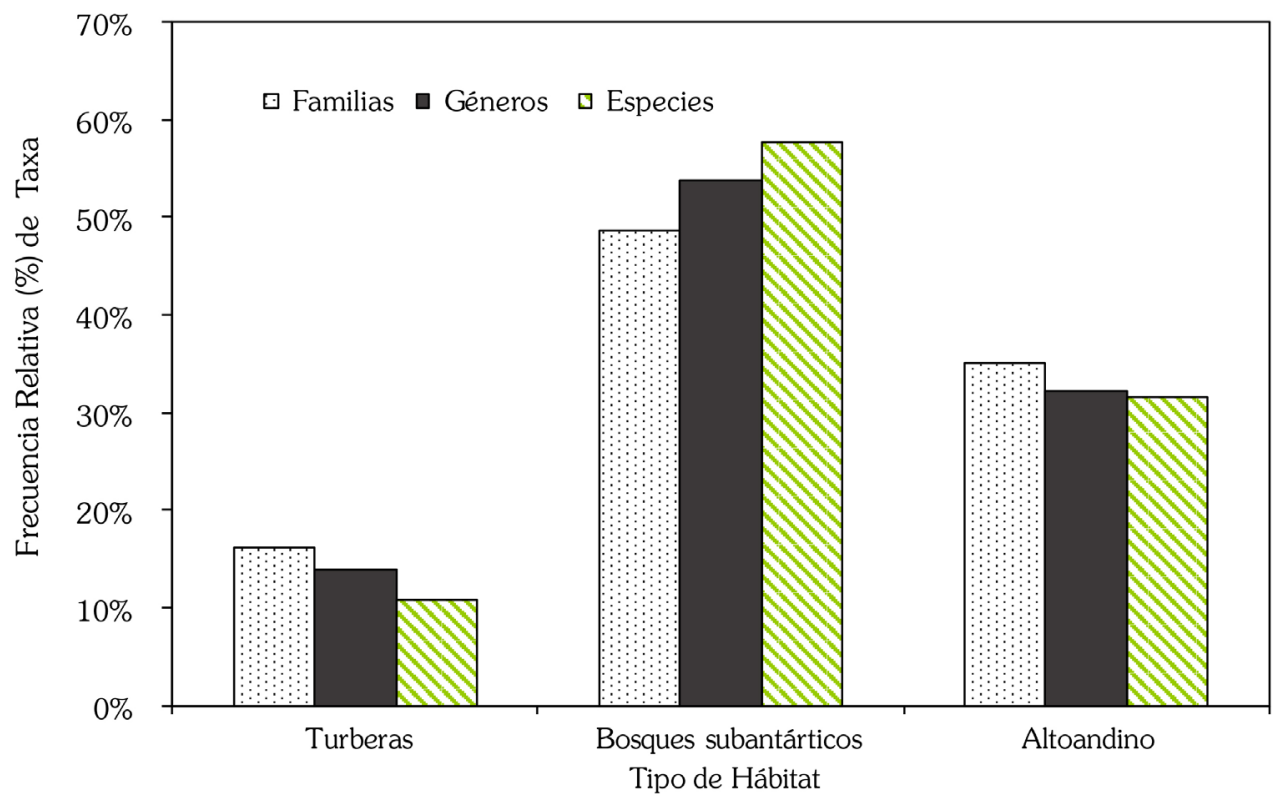

Fig. 2. Riqueza relativa de especies, géneros y familias de musgos por tipo de hábitat en la Isla Navarino. Datos tomados de Buck \& Goffinet (2010), Goffinet et al. (2012), Rozzi et al. (2012a), Méndez et al. (2013).

otras dos especies de Sphagnum que crecen por sobre el nivel de las aguas, y en consecuencia son más fáciles de detectar. Aunque su presencia se ha documentado para otros sectores de la ecorregión subantártica de Magallanes (Díaz et al. 2005), S. falcatulum está pobremente registrado en la Isla Navarino, detectado el año 2013 en la zona de Puerto Toro (al Este) a un costado de un camino vehicular e ingresada a la colección del New York Botanical Garden (http://sweetgum.nybg.org/ science/vh/). De acuerdo con Karlin et al. (2013), $S$. falcatulum es la especie de Sphagnum más ampliamente distribuida en el Holoantártico (que incluye el continente Antártico, la Tierra del Fuego, la ecorregión subantártica de Magallanes, Nueva Zelanda, y las islas del océano Antártico al sur de los $40^{\circ}$ latitud).

La singularidad del hábitat donde registramos a S. falcatulum -ilustrado en las fotografías de la Figs. 3A, B y C- y las diferencias ecológicas que esto supone con sus especies hermanas $S$. magellanicum y $S$. fimbriatum más abundantes en la zona norte de la Isla Navarino, demuestran que la observación en terreno es fundamental para registrar taxa poco percibidos que permanecen invisibles para la ciencia, la educación, la conservación y posibles usos sustentables, tales como el turismo de intereses especiales. El Ministerio de Bienes Nacionales ha promovido la implementación de rutas patrimoniales, de las cuales forma parte la ruta Cabo de Hornos: Lago Windhond (http://rutas.bienes.cl/?p=858), y por tanto es fundamental contribuir a que los diferentes tipos de hábitats de la heterogénea ecorregión subantártica de Magallanes (cfr. Rozzi et al. 2012b) queden representados para facilitar a los visitantes la observación y aprecio de la brioflora en estos lugares remotos y prístinos en la Reserva de Biosfera Cabo de Hornos.

Esta nota anima al lector, ya sea científico o aficionado a las ciencias naturales, a observar, descubrir y comprender el entorno que le rodea. La experiencia directa en el ambiente natural es indispensable para la sensibilización de la población crecientemente urbana. La ética biocultural propone que el ecoturismo ofrece experiencias de comprensión holística de las relaciones entre los hábitats de una región y los hábitos de vida de sus diversos co-habitantes, incluyendo la diminuta flora de briófitas (Rozzi, 2013). Las observaciones y experiencias en el campo han sido centrales en la tradición naturalista que dio origen a la enseñanza 

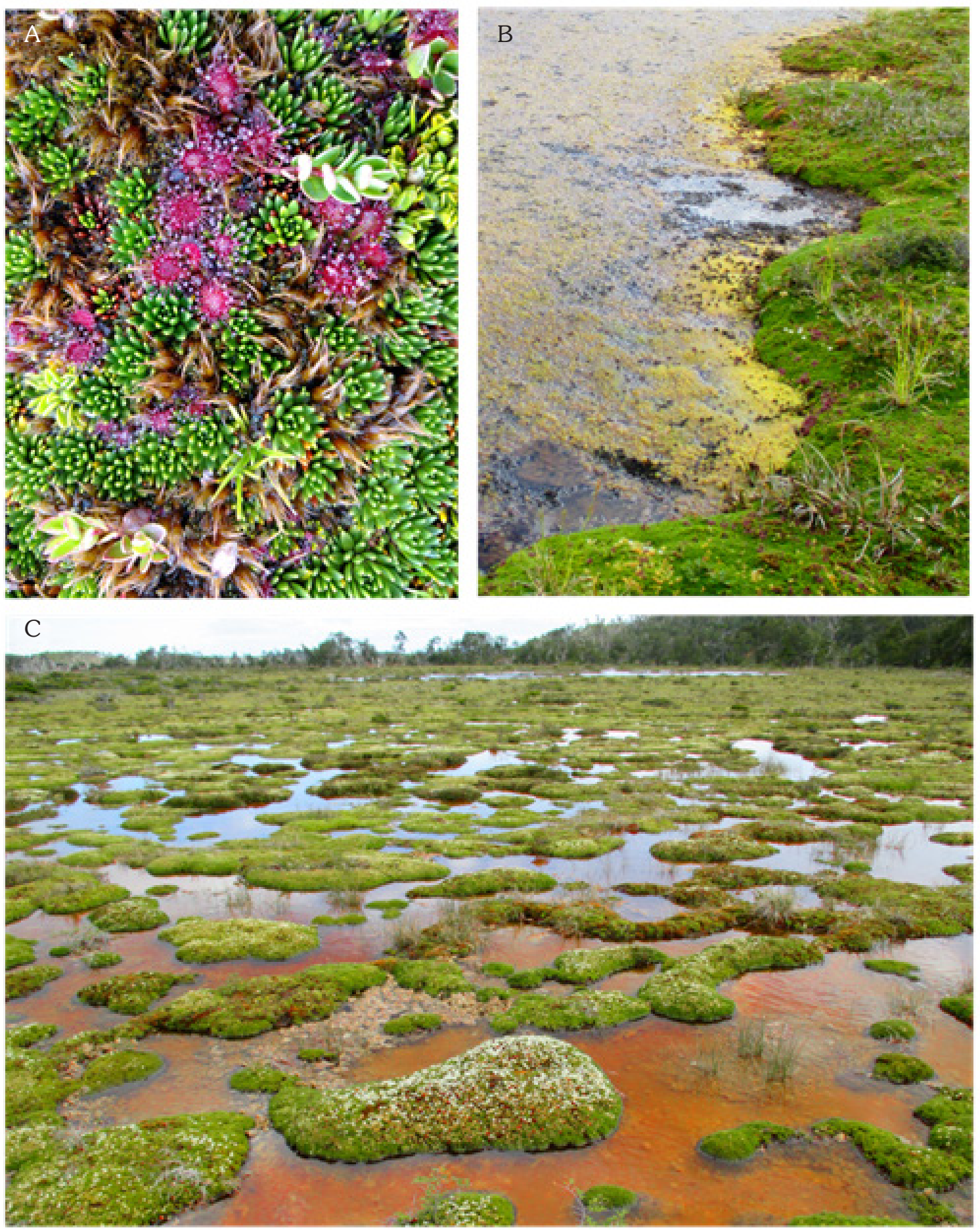

Fig. 3. Complejo de tunda de Magallanes, incluye plantas en cojín en los alrededores de lago Windhond en el sector sur de la Isla Navarino $\left(55^{\circ} 05,858^{\prime} \mathrm{S}, 67^{\circ} 36,594^{\prime} \mathrm{W}\right)$ donde se realizó el nuevo registro de Sphagnum falcatulum. A: Acercamiento de una planta carnívora Drosera uniflora, creciendo sobre un cojín de Donatia fascicularis. B: Vista del tipo de hábitat configurado por un mosaico de plantas en cojín y pozas con sedimentos de turba . C: Margen de una poza en donde se registró una abundante población de S. falcatulum (amarillo), sumergida en el agua. Fotografías de R. Mackenzie y L. Lewis. 
de las ciencias biológicas mediante el método científico, y es esencial para que los estudiantes y visitantes comprendan la complejidad espacial y temporal de los ecosistemas (Willson \& Armesto, 2006; Villagrán et al. 2014). Para esto, el Programa de Conservación Biocultural de la Universidad de Magallanes y el Instituto de Ecología y Biodiversidad en colaboración con la University of North Texas (EE.UU.) han organizado desde hace más de diez años consecutivos el curso internacional de Filosofía Ambiental de Campo (Rozzi et al. 2008b), en el cual estudiantes de diferentes disciplinas -ligadas o no a las ciencias biológicas-, tienen la oportunidad de experimentar e integrar la ecología y la ética ambiental observando directamente los fenómenos en su entorno natural.

El nuevo registro de Sphagnum falcatulum es resultado de esta actividad y constituye un hallazgo significativo para la ciencia, la conservación y el ecoturismo de esta Reserva de la Biosfera. Para la ciencia, señala la importancia de continuar estudios florísticos en hábitats menos estudiados, como el complejo de tundra de Magallanes. Para la conservación demuestra la compleja biodiversidad del complejo de tundra, que es tratado en forma gruesa como recurso minero por la ley chilena (Domínguez et al. 2012). Para el turismo, nuestro registro de $S$. falcatulum demuestra las singularidades que pueden observar los visitantes que tengan la experiencia de ecoturismo con lupa. Para alcanzar este objetivo es fundamental trasferir los resultados de la investigación científica a la educación e implementación de senderos interpretativos y rutas patrimoniales que permitan apreciar la belleza y valorar la existencia de una comunidad de pequeños cohabitantes que presentan hábitos de vida únicos en los singulares hábitats de la Reserva de la Biosfera Cabo de Hornos.

\section{AGRADECIMIENTOS}

Agradecemos a las numerosas personas $e$ instituciones que han colaborado con los cursos de Filosofía Ambiental de Campo desarrollados en el Parque Etnobotánico Omora desde el año 2005. A Sanja Fistric del Centro de Estudios Ambientales UFZ, Leipzig, Alemania, y Rosa María Gallardo del Centro de Energías Renovables (CERE),
Universidad de Magallanes (UMAG) por su análisis de las imágenes satelitales. Este trabajo contó con apoyo del Laboratorio de Biología Molecular y Biotecnología Vegetal de la P. Universidad Católica de Chile y de los proyectos ICM P05002 (MINECON) y Basal-CONICYT PFB-23 del Instituto de Ecología y Biodiversidad de Chile (IEB). Este artículo es una contribución del Programa de Conservación Biocultural Subantártica, coordinado por UMAG e IEB en colaboración con University of North Texas, EE.UU.

\section{LITERATURA CITADA}

Buck, W. R., \& B. Goffinet. (2010). Peliminary key to the mosses of Isla Navarino, Chile (Prov. Antártica Chilena). Nova Hedwigia, 138,215-229.

Díaz, M. F., Larraín, J., \& Zegers, G. (2005). Guía para el conocimiento de la flora de turberas y pomponales de la Isla Grande de Chiloé. Informe Fundación Senda Darwin: http:// www.sendadarwin.cl/espanol//?page_ $\mathrm{id}=431$

Domínguez, E., \& Vega-Valdés, D. (Eds.). (2015). Funciones y servicios ecosistémicos de las turberas en Magallanes. Colección de libros INIA $\mathrm{N}^{\circ}$ 33. Instituto de Investigaciones Agropecuarias. Centro Regional de Investigación Kampenaike. Punta Arenas, Chile.

Domínguez, E., Bahamonde, N., \& MuñozEscobar, C. (2012). Efectos de la extracción de turba sobre la composición y estructura de una turbera de Sphagnum explotada y abandonada hace 20 años, Chile. Anales del Instituto de la Patagonia, 40(2),37-45. Goffinet, B., Rozzi, R., Lewis, L., Buck, W., \& Massardo, F. (2012). Los Bosques en Miniatura del Cabo de Hornos: Ecoturismo con Lupa. Denton y Punta Arenas. UNT Press-Ediciones y Universidad de Magallanes.

Karlin, E. F., Buck, W. R., Seppelt, R. D., Boles, S. B., \& Shaw, J. (2013). The double allopolyploid Sphagnum $\times$ falcatulum (Sphagnaceae) in Tierra del Fuego, a Holantarctic perspective. Journal of Bryology, 35(3), 157-172. 
Kostka, J. E., Weston, D. J., Glass, J. B., Lilleskov, E. A., Shaw, A. J., \& Turetsky, M. R. (2016). The Sphagnum microbiome: new insights from an ancient plant lineage. New Phytologist 210, DOI: 10.1111/ nph.13993.

Méndez, M., Rozzi, R., \& Cavieres, L. (2013). Flora vascular y no-vascular en la zona altoandina

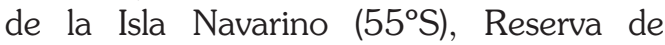
biosfera Cabo de Hornos, Chile. Gayana Botánica, 70, 337-343.

Mittermeier, R. A., Mittermeier, C. G., Brooks, T. M., Pilgrim, J. D., Konstant, W. R., da Fonseca, G. A. B., \& Kormos, C. (2003). Wilderness and biodiversity conservation. Proceedings of the National Academy of Sciences , 100, 10309-10313.

Pisano, E. (1977). Fitogeografía de FuegoPatagonia Chilena. I. Comunidades vegetales entre las latitudes $52^{\circ}$ y $56^{\circ} \mathrm{S}$. Anales del Instituto de la Patagonia, 8,121-250.

Pisano, E. (1980). Distribución y características de la vegetación del archipiélago del Cabo de Hornos. Anales del Instituto de la Patagonia, 11, 191-224.

Pisano, E. (1992). Sectorización fitogeográfica del archipiélago Sud Patagónico-Fueguino V. Sintaxonomía y distribución de las unidades de vegetación vascular. Anales del Instituto de la Patagonia, Serie Ciencias Naturales, 21, 5-33.

Rozzi, R. (2013). Biocultural Ethics: From Biocultural Homogenization toward Biocultural Conservation. In R. Rozzi, S. T. A. Pickett, C. Palmer, J. J. Armesto \& J. B. Callicott (Eds.), Linking Ecology and Ethics for a Changing World: Values, Philosophy, and Action. Ecology and Ethics (pp. 9-32). Dodrecht: Springer.

Rozzi, R., Arango, X., Massardo, F., Anderson, C., Heidinger, K., \& Moses, K.P. (2008b). Field environmental philosophy and biocultural conservation: The Omora Ethnobotanical Park educational program. Environmental Ethics, 30, 325-336.
Rozzi, R., Armesto, J. J., Goffinet, B., Buck, W., Massardo, F., \& Callicott, J. B. (2008a). Changing lenses to assess biodiversity: Patterns of species richness in subAntarctic plants and implications for global conservation. Frontiers in Ecology and the Environment, 6(3), 131-137.

Rozzi, R., Armesto, J. J., Gutiérrez, J., Massardo, F., Likens, G.,...Kalin, M. T. (2012b). Integrating ecology and environmental ethics: Earth stewardship in the southern end of the Americas. BioScience, 62, 226236.

Rozzi, R., Lewis, L., Massardo, F., Medina, Y., Moses, K.,...Goffinet, B. (2012a). Ecoturismo con Lupa en el Parque Omora. Punta Arenas. Ediciones Universidad de Magallanes.

Rozzi, R., Massardo, F., Anderson, C. B., Heidinger, K., \& Silander, J. A. (2006). Ten principles for biocultural conservation at the southern tip of the Americas: the approach of the Omora Ethnobotanical Park. Ecology and Society, 11, 43-70.

Rozzi, R., Massardo, F., Mansilla, A., Anderson, C. B., Berghöfer, A., Barros, E. (2007). La Reserva De Biosfera Cabo De Hornos: Un Desafío para la Conservación de la Biodiversidad e Implementación Del Desarrollo Sustentable en el Extremo Austral de América. Anales del Instituto de la Patagonia, 35(1), 55-70.

Van Breemen, N. 1995. How Sphagnum bogs down other plants. Trends in Ecology and Evolution, 10, 270-275.

Villagrán, C., Segovia, R., \& Castillo, L. (2014). Principios de la investigación en ciencias naturales históricas: ¿ Por qué en biología es necesaria la Historia Natural de los organismos?. Gayana Botánica, 71(2), 259-266.

Willson, M. F., \& Armesto, J. J. (2006). Is natural history really dead? Toward the rebirth of natural history. Revista Chilena de Historia Natural, 79, 279-283. 